\title{
AN EXISTENCE TIME ESTIMATE FOR KÄHLER-RICCI FLOW
}

\author{
ALBERT CHAU ${ }^{1}$, KA-FAI LI, AND LUEN-FAI TAM ${ }^{2}$
}

\begin{abstract}
Fix a complete noncompact Kähler manifold $\left(M^{n}, h_{0}\right)$ with bounded curvature. Let $g(t)$ be a bounded curvature solution to the Kähler-Ricci flow starting from some $g_{0}$ which is uniformly equivalent to $h_{0}$. We estimate the existence time of $g(t)$ together with $C^{0}$ bounds and curvature bounds, where the estimates depend only on $h_{0}$ and the $C^{0}$ distance between $g_{0}$ and $h_{0}$. We also generalize these results to cases when $g_{0}$ may have unbounded curvature.

Keywords: Kähler-Ricci flow, existence time
\end{abstract}

\section{INTRODUCTION}

A motivation of this work is to understand the Kähler-Ricci flow on complete noncompact Kähler manifolds with initial metrics which may not have bounded curvature. The Kähler Ricci flow is the following evolution equation for Kähler metric $g_{0}$ on an $n$ dimensional complex manifold $M^{n}$

$$
\left\{\begin{array}{l}
\frac{\partial g_{i \bar{\jmath}}}{\partial t}=-R_{i \bar{\jmath}} \\
g(0)=g_{0} .
\end{array}\right.
$$

When $g_{0}$ is complete with curvature bounded by $k_{0}$, Shi showed in [8] that (1.1) has a short time solution $g(t)$ on $M \times[0, T)$ having bounded curvature for all $t$ (see Theorem A.1). Moreover, $T$ and the curvature bound of $g(t)$ can be estimated respectively in terms on $n, k_{0}$ and $n, k_{0}, t$.

A fundamental problem is to determine the maximal such $T$ for a given $g_{0}$, and we will denote this time by $T_{g_{0}}$. Extending the result of Tian-Zhang [9] for the compact case, Lott-Zhang gave an analytic

\footnotetext{
${ }^{1}$ Research partially supported by NSERC grant no. \#327637-06.

${ }^{2}$ Research partially supported by Hong Kong RGC General Research Fund \#CUHK 14305114 .

2000 Mathematics Subject Classification. Primary 53C55, 58J35.
} 
characterization of $T_{g_{0}}$ for general complete Kähler manifolds (see Theorem 2.1) which in particular extended Shi's result above. In this work we want to estimate $T_{g_{0}}$ in terms of $C^{0}$ norm of $g_{0}$. Firstly we will use Theorem 2.1 to show that:

$$
C_{1} h_{0} \leq g_{0} \leq C_{2} h_{0} \quad \Longrightarrow \quad C_{1} T_{h_{0}} \leq T_{g_{0}} \leq C_{2} T_{h_{0}}
$$

where $C_{i}>0$ are constants and $g_{0}, h_{0}$ are complete Kähler metrics with bounded curvature (see Theorem [2.2). As a consequence, $T_{g}$ is continuous on the space of Kähler metrics on $M$ in the $C^{0}$ norm. The usefulness of (1.2) comes when considering the flow of an arbitrary metric $g_{0}$ which is uniformly equivalent to the initial condition $h_{0}$ of a known solution $h(t)$, in which case Theorem 2.2 tells us the existence time for $g(t)$ is uniformly comparable to that of $h(t)$. In particular, if $h(t)$ exists for all time, then so does the flow of any bounded curvature Kähler metric which is equivalent to $h_{0}$. For example, one can show that if $g_{0}$ has bounded curvature and $g_{0}$ is uniformly equivalent to a complete Kähler metric $h_{0}$ with bounded curvature so that the $\operatorname{Ric}\left(h_{0}\right) \leq 0$, then $T_{g_{0}}=\infty$.

In the above context, one may also ask about a priori estimates for the solution $g(t)$ in terms of the known solution $h(t)$. In the context of (1.2) we will prove that: for any $0<S<C_{1} T_{h_{0}}$ we have

$$
C_{n, S, h_{0}, C_{1}, C_{2}, \Lambda}^{-1} h_{0} \leq g(t) \leq C_{n, S, h_{0}, C_{1}, C_{2}, \Lambda} h_{0} \quad \text { on } M \times[0, S]
$$

where $\Lambda=\sup _{M}\left\|R m\left(g_{0}\right)\right\|_{g_{0}}$. On the other hand, by restricting the time interval we can actually remove the dependence on the curvature of $g_{0}$ : for any $0<s<S<\frac{C_{1}}{n} T_{h_{0}}$ we have

$$
C_{n, s, S, h_{0}, C_{1}, C_{2}}^{-1} h_{0} \leq g(t) \leq C_{n, s, S, h_{0}, C_{1}, C_{2}} h_{0} \quad \text { on } M \times[s, S]
$$

See Theorem 2.3 for these estimates. As mentioned above in [8] Shi proved the estimates in (1.3), but with the upper time limit $C_{1} T_{h_{0}}$ replaced by some $T(n, \Lambda)$ depending only on $n, \Lambda$ (in this case, as in [8], one may just take $\left.h_{0}=g_{0}\right)$. The point is that in many cases, $C_{1} T_{h_{0}}$ will be strictly larger than $T(n, \Lambda)$, thus leading to a better estimate of $T_{g_{0}}$ and corresponding estimates.

From (1.2) and (1.3) we may then conclude higher order estimates as in Theorem 2.4. One can then apply these estimates to study KählerRicci flow with initial metric which may have unbounded curvature and existence of solutions $g(t)$ starting from $C^{0}$ limits of bounded curvature metrics uniformly equivalent to some fixed $h_{0}$ with bounded curvature (Theorem 3.1). This generalizes some of our earlier results in [1]. 
The paper is organized as follows. In $\S 2.1$ we prove the existence time comparison in (1.2). In $\S 2.2$ we establish the comparison in (1.3), then establish the corresponding higher order estimates (1.3), (1.4) in $\S 2.3$. In $\S 3$ we generalize to initial metrics $g_{0}$ which may only be Hermitian continuous. Finally we collect some known results, which we will use throughout, in the Appendix.

\section{EXISTENCE TIME AND ESTIMATES}

Suppose $\left(M^{n}, g_{0}\right)$ is a complete noncompact Kähler manifold with bounded curvature, and let $g(t)$ be a solution to Kähler-Ricci flow (1.1) on $M \times[0, T)$ with $g(0)=g_{0}$. We make the following:

Definition 2.1. We call the solution $g(t)$

(1) a bounded curvature solution starting from $g_{0}$ if $g(t)$ has uniformly bounded curvature on $M \times[0, S]$ for all $S<T$;

(2) the maximal bounded curvature solution starting from $g_{0}$ if $g(t)$ satisfies the condition in (1) and $T$ is maximal with this property, in which case we denote $T=T_{g_{0}}$.

By Theorem A.3, a bounded curvature solution starting from $g_{0}$, on $M \times[0, T)$, is indeed the unique solution with bounded curvature.

Now let $\left(M^{n}, h_{0}\right)$ be a fixed complete noncompact Kähler manifold with bounded curvature. We define the following spaces of Kähler metrics.

Definition 2.2. For any $0<a<b$ and $\Lambda>0$, we define the sets

$$
\mathcal{G}\left(h_{0} ; a, b, \Lambda\right) \subset \mathcal{G}\left(h_{0} ; a, b\right) \subset \mathcal{G}\left(h_{0} ; a\right)
$$

as follow

(1) $\mathcal{G}\left(h_{0} ; a\right)$ is the set of bounded curvature Kähler metrics $g_{0}$ on $M$ such that $a h_{0} \leq g_{0} \leq C\left(g_{0}\right) h_{0}$ for some constant $C\left(g_{0}\right)$ which may depend on $g_{0}$

(2) $\mathcal{G}\left(h_{0} ; a, b\right)$ is the set of bounded curvature Kähler metrics $g_{0}$ on $M$ such that $a h_{0} \leq g_{0} \leq b h_{0}$

(3) $\mathcal{G}\left(h_{0} ; a, b, \Lambda\right)$ is the set of Kähler metrics $g_{0}$ on $M$ with sectional curvatures bounded by $\Lambda$ and $a h_{0} \leq g_{0} \leq b h_{0}$

For $g_{0}$ in each of the above classes, we want to estimate $T_{g_{0}}$ and various bounds on the corresponding maximal bounded curvature solution. First we need the following result by Lott-Zhang [6].

Theorem 2.1. (Lott-Zhang [6]) Let $g_{0}$ be a complete Kähler metric with bounded curvature on a non-compact manifold $M$. Then $T_{g_{0}}$ is equal to the supremum of the numbers $T$ for which there is a bounded function $F_{T} \in C^{\infty}(M)$ such that 
(i) $g_{0}-T \operatorname{Ric}\left(g_{0}\right)+\sqrt{-1} \partial \bar{\partial} F_{T} \geq c_{T} g_{0}$ for some $c_{T}>0$

(ii) $\left|F_{T}\right|$ and the quantities $\left|\nabla^{l} \operatorname{Rm}\left(g_{0}\right)\right|_{g_{0}},\left|\nabla^{l} \partial \bar{\partial} F_{T}\right|_{g_{0}}$, for $0 \leq l \leq 2$, are uniformly bounded on $M$.

Moreover if $T$ satisfies ( $i$ ) and (ii), then for any $T^{\prime}<T$, there is a constant $C$ depending $T^{\prime}, c_{T}$, and the bound on the quantities in (ii) such that

$$
C^{-1} g_{0} \leq g(t) \leq C g_{0}
$$

on $M \times\left[0, T^{\prime}\right]$.

Proof. Suppose the above conditions are satisfied for some $T<\infty$. If $0<T<T_{g_{0}}$ then the proof Theorem 4.1 in [6] shows the existence of a bounded $F_{T}$ which satisfies (i) and also has bounded covariant derivatives of all orders, thus also satisfying (ii). Conversely, given $F_{T}$ satisfying (i) and (ii), by the estimates in the proof of [6, Theorem 4.1] (up to and including Lemma 4.26) there exists constants $C_{1}, C_{2}>0$ such that $C_{1} g \leq g(t) \leq C_{2} g$ for all $t \in\left[0, \min \left(T_{g_{0}}, T\right)\right)$. Then by Theorem A.2. we have that the curvature of $g(t)$ is uniformly bounded on $M \times\left[0, \min \left(T_{g_{0}}, T\right)\right)$ which implies $0<T<T_{g_{0}}$ by Theorem A.1. This completes the proof that $T_{g}$ is characterized by (i) and (ii).

Moreover, from the above mentioned estimates in [6], one can see that the last statement in the Theorem also holds.

2.1. existence time estimate. The first result of this section is the following existence time estimate:

Theorem 2.2. $T_{g_{0}} \geq a T_{h_{0}}$ for all $g_{0} \in \mathcal{G}\left(h_{0} ; a\right)$.

Proof. First observe that if $\lambda>0$ is a constant, Rethen $T_{\lambda h_{0}}=\lambda T_{h_{0}}$. Hence without loss of generality, we may assume that $a=1$. Also, we assume without loss of generality that $g_{0}, h_{0}$ have bounded geometry of order $\infty$. For if not, we let $g(t), h(t)$ are the corresponding solutions as in Theorem A.1, then we first prove the Theorem for $g(\epsilon), h(\epsilon)$ for arbitrary small $\epsilon$ and then let $\epsilon \rightarrow 0$ and use the fact that $g(\epsilon), h(\epsilon)$ converge uniformly to $g_{0}, h_{0}$ (respectively) on $M$ by Corollary A.1, and $T_{g(\epsilon)}=T_{g}-\epsilon$ and $T_{h(\epsilon)}=T_{h}-\epsilon$ by Theorem A.3. Note that by Theorem A.2, all the covariant derivatives of $g(\epsilon)$ with respect to $h_{0}$ are bounded. So we may assume in addition that all the covariant derivatives of $g_{0}$ with respect to $h_{0}$ are bounded.

Now for any $0<T<T_{h_{0}}$, we have for $g_{0} \in \mathcal{G}\left(h_{0} ; a\right)$,

$$
g_{0}-T \operatorname{Ric}\left(g_{0}\right)=h_{0}-T \operatorname{Ric}\left(h_{0}\right)+T\left(\operatorname{Ric}\left(g_{0}\right)-\operatorname{Ric}\left(h_{0}\right)\right)+\left(g_{0}-h_{0}\right) .
$$


By Theorem 2.1, there is a smooth bounded function $f$ with bounded covariant derivatives with respect to $h_{0}$ such that

$$
h_{0}-T \operatorname{Ric}\left(h_{0}\right)+\sqrt{-1} \partial \bar{\partial} f \geq C_{1} h_{0}
$$

for some $C_{1}>0$. Then letting $F=\log \frac{\omega_{0}^{n}}{\eta^{n}}$ where $\omega_{0}$ and $\eta$ are the Kähler forms of $g_{0}$ and $h_{0}$ respectively, gives

$$
\begin{aligned}
g_{0}-T \operatorname{Ric}\left(g_{0}\right)+\sqrt{-1} \partial \bar{\partial}(f+T F) & \geq C_{1} h_{0}+\left(g_{0}-h_{0}\right) \\
& \geq C_{2} g_{0}
\end{aligned}
$$

for some $C_{2}>0$ because $g_{0} \geq h_{0}$ and $g_{0}$ is uniformly equivalent to $h_{0}$. From the facts that $g_{0}, h_{0}$ are equivalent and that all the covariant derivatives of $g_{0}$ with respect to $h_{0}$ are bounded, we may conclude that all the covariant derivatives of $f$ are bounded with respect to $g_{0}$ as well. We may also conclude from these facts that $F$ and $\left|\nabla^{l} \partial \bar{\partial} F\right|_{g_{0}}$ are uniformly bounded for $0 \leq l \leq 2$ where we have used that $\sqrt{-1} \partial \bar{\partial} F=$ $-\operatorname{Ric}\left(g_{0}\right)+\operatorname{Ric}\left(h_{0}\right)$. By Theorem 2.1, we conclude that $T \leq T_{g_{0}}$. From this the result follows.

By the theorem, we have the following monotonicity and continuity of $T_{g}$. Namely

Corollary 2.1. Let $M^{n}$ be a noncompact complex manifold.

(i) Let $g_{0} \geq h_{0}$ be complete uniformly equivalent Kähler metrics on $M$ with bounded curvature. Then $T_{g_{0}} \geq T_{h_{0}}$. In particular, if $T_{h_{0}}=\infty$, then $T_{g_{0}}=\infty$.

(ii) Let $\mathcal{K}$ be the set of complete Kähler metrics on $M$ with bounded curvature. Then $T_{g}$ is continuous on $\mathcal{K}$ with respect to the $C^{0}$ norm in the following sense: Let $g_{0} \in \mathcal{K}$. Then for $h_{0} \in \mathcal{K}$, $T_{h_{0}} \rightarrow T_{g_{0}}$ as $\left\|h_{0}-g_{0}\right\|_{g_{0}} \rightarrow 0$.

We can extend the existence time estimate in Corollary 4.2 [1]. Namely, we can estimate the existence time in terms of upper bound of Ricci curvature instead of upper bound of the bisectional curvature.

Corollary 2.2. Let $(M, h)$ be a complete Kähler metric having bounded curvature with Ricci curvature bounded above by $K$, and let $g \in \mathcal{G}(h ; 1)$. then $T_{g} \geq \frac{1}{K}$.

Proof. Since $\operatorname{Ric}(h) \leq K h$, so $h-t \operatorname{Ric}(h)=(1-K t) h$. By Theorem 2.1, $T_{h} \geq \frac{1}{K}$. By Theorem 2.2, $T_{g} \geq \frac{1}{K}$. 
2.2. $C^{0}$ estimates. In this section we will establish $C^{0}$ bounds for maximal solutions $g(t)$ starting from some $g_{0} \in \mathcal{G}\left(h_{0} ; a, b\right)$, where the bounds are uniform over all such $g_{0}$. We will establish similar bounds for maximal solutions $g(t)$ starting from some $g_{0} \in \mathcal{G}\left(h_{0} ; a, b, \Lambda\right)$. We begin with the following:

Lemma 2.1. Let $g_{0}$ and $h_{0}$ be complete noncompact Kähler metrics on $M^{n}$ with curvature bounded by $k_{0}$ such that $A^{-1} g_{0} \leq h_{0} \leq A g_{0}$. Let $T\left(n, k_{0}\right)>0$ be the existence time of Kähler-Ricci flows with initial data $g_{0}, h_{0}$ in Theorem A.1. For any $0<T<T\left(n, k_{0}\right)$, and for any tensor $\Theta$ on $M$. Suppose all the covariant derivatives of $\Theta$ with respect to $h(T)$ are bound. Then the covariant derivatives of $\Theta$ with respect to $g(T)$ are also bounded. Namely, for any $l \geq 0$

$$
\left|\nabla_{g(T)}^{l} \Theta\right|_{g(T)} \leq D_{l}
$$

where $D_{l}$ is a constant depending only on $k_{0}, n, T, A, l$ and the bounds of $\left|\nabla_{h(T)}^{k} \Theta\right|_{h(T)}$ with $0 \leq k \leq l$.

Proof. By Theorem A.1, the curvature and all the covariant derivatives up to order $l$ of both $h(t), g(t)$ are uniformly bounded on $M \times\left[\frac{T}{2}, T\right]$ by a constant $C_{1}$ depending only on $n, k_{0}, T, l$. Hence by the KählerRicci flow equations, we have $C_{2}^{-1} h(T) \leq g(t) \leq C_{2} h(T)$ for $t \in\left[\frac{T}{2}, T\right]$, where $C_{2}$ is a constant depending only on $n, k_{0}, T$. By Theorem A.2, we conclude that

$$
\left|\nabla_{h(T)}^{k} g(T)\right|_{h(T)} \leq C_{3}
$$

for all $0 \leq k \leq l$ for some constant $C_{3}$ only on $n, k_{0}, T, l$. From this, it is easy to see the lemma is true.

Theorem 2.3. Let $h_{0}$ be as before.

(1) For any $0<a<b$ and $\Lambda>0$, and any $0<S<a T_{h_{0}}$ there is $a$ constant $C$ depending only on $n, S, h_{0}, a, b, \Lambda$ such that

$$
C^{-1} h_{0} \leq g(t) \leq C h_{0}
$$

on $M \times[0, S]$ for all maximal solutions $g(t)$ starting from some $g_{0} \in \mathcal{G}\left(h_{0} ; a, b, \Lambda\right)$.

(2) For any $0<a<b$, and any $0<S<\frac{a}{n} T_{h_{0}}$ there is a constant $C$ depending only on $n, s, S, h_{0}, a, b$ such that

$$
C^{-1} h_{0} \leq g(t) \leq C h_{0}
$$

on $M \times[0, S]$ for all maximal solutions $g(t)$ starting from some $g_{0} \in \mathcal{G}\left(h_{0} ; a, b\right)$. 
Proof. As before, we may assume that $a=1$. We first prove part (1). Let $h(t)$ be the maximal solution starting from $h_{0}$ and let $g(t)$ be the maximal solution $g(t)$ starting from some $g_{0} \in \mathcal{G}\left(h_{0} ; a, b, \Lambda\right)$. Let $0<S<T_{h_{0}}$ be given. Fix $\alpha<1$ such that $\alpha T_{h_{0}}>S$. Then $T_{\alpha h_{0}}=\alpha T_{h_{0}}>S$. We claim that there is $\epsilon>0$ depending only on $n, S, h_{0}, a, b, \Lambda$ with $S<\alpha T_{h_{0}}-\epsilon$ and satisfies the following:

(i) $C^{-1} h_{0} \leq g(t) \leq C h_{0}$ for all $t \leq \epsilon$ and some $C>0$ depending only on $n, \Lambda$;

(ii) $\hat{h}(t)=\alpha h\left(\alpha^{-1}(t+\epsilon)\right)$ is the solution the Kähler-Ricci flow equation on $M \times[0, S]$ with initial data $\hat{h}(0)=\alpha h\left(\alpha^{-1} \epsilon\right)$ such that

$$
2 b \alpha^{-1} \hat{h}(0)=2 b h\left(\alpha^{-1} \epsilon\right) \geq g(\epsilon)>\alpha h\left(\alpha^{-1} \epsilon\right)=\hat{h}(0) ;
$$

(iii) and there exists a constant $C_{1}>0$ and a smooth function $f$ with

$$
\hat{h}(0)-\operatorname{SRic}(\hat{h}(0))+\sqrt{-1} \partial \bar{\partial} f \geq C_{1} \hat{h}(0)
$$

where $C_{1}$ and the covariant derivatives of $f$ with respect to $h\left(\frac{\epsilon}{\alpha}\right)$ are bounded by constants depending only on $n, h_{0}, \epsilon, \alpha$.

Indeed, by Theorem A.1 and Corollary A.1 we may choose $\epsilon$ depending only on $n$ and $\Lambda$ so that (i) and (ii) hold because $S<\alpha T_{h_{0}}-\epsilon=$ $T_{\hat{h}(0)}$. (iii) holds for some $C_{1}, f$ by Theorem 2.1 applied to the solution $\hat{h}(t)$.

Then letting $F=\log \frac{\operatorname{det}\left(\alpha h\left(\alpha^{-1} \epsilon\right)\right)}{\operatorname{det}(g(\epsilon))}$ and using (ii) and (iii)

$$
g(\epsilon)-S \operatorname{Ric}(g(\epsilon))+\sqrt{-1} \partial \bar{\partial}(f+S F) \geq C_{2} g(\epsilon)
$$

for some $C_{2}>0$ depending only on $\epsilon, a, b, \Lambda$. Now $\left\|\nabla^{l} \operatorname{Rm}(g(\epsilon))\right\|_{g(\epsilon)}$ and $\|\left.\nabla^{l} \operatorname{Rm}(h(\epsilon))\right|_{h(\epsilon)}$ are bounded by constants depending only on $n, h_{0}, S, a, b, \Lambda$. Using (i) and Lemma 2.1, we may conclude that the covariant derivatives of $(f+S F)$ with respect to $g(\epsilon)$ are all bounded by constants depending only on $n, h_{0}, \Lambda, S, a, b$. From this and (2.1), the last statement in Theorem 2.1 gives $C^{-1} h_{0} \leq \hat{g}(t+\epsilon) \leq C h_{0}$ for all $t \leq S$ and some $C$ depending only on $n, h_{0}, S, a, b, \Lambda$. We conclude from this and (i) that (1) is true. This completes the proof of (1).

To prove (2), by [1] if $g(t)$ is the Kähler-Ricci flow with bounded curvature so that $g(0)=g_{0} \in \mathcal{G}\left(h_{0} ; 1, b\right)$, then

$$
\left(\frac{1}{n}-2 K t\right) h_{0} \leq g(t) \leq B(t) h_{0}
$$

on $M \times\left[0, \frac{1}{2 n K}\right)$ for some continuous function $B(t)>0$ on $\left[0, \frac{1}{2 n K}\right)$ which depends only on $n, K$. Here $K \geq 0$ is an upper bound for the 
bisectional curvature of $h_{0}$. By Theorem A.2 and A.1, one can see that the (2) follows from (1), by considering $g(\epsilon)$ for $\epsilon>0$ small.

2.3. higher order estimates. By the local Evans-Krylov Theory in [3, 5, 7], more precisely by Theorem A.2, we may have the following estimates based on the $C^{0}$ estimates in Theorem 2.3.

Theorem 2.4. Let $h_{0}$ be as before.

(1) For any $0<S<a T_{h_{0}}$ and for all $l \geq 0$, there is a constant $C_{l}$ depending only on $n, S, h_{0}, a, b, \Lambda$ and $l$.

$$
\sup _{M \times[0, S]}\left\|\nabla_{g(t)}^{l} \operatorname{Rm}(g(t))\right\|_{g(t)}^{2} \leq \frac{C_{l}}{t^{l}}
$$

for all maximal solutions $g(t)$ starting from some $g_{0} \in \mathcal{G}\left(h_{0} ; a, b, \Lambda\right)$.

(2) For any $0<s<S<\frac{a}{n} T_{h_{0}}$ there is a constant $C$ depending only on $n, s, S, h_{0}, a, b$ such that

$$
\sup _{M \times[s, S]}\left\|\nabla_{g(t)}^{l} \operatorname{Rm}(g(t))\right\|_{g(t)}^{2} \leq \frac{C}{t^{l+2}}
$$

for all maximal solutions $g(t)$ starting from some $g_{0} \in \mathcal{G}\left(h_{0} ; a, b\right)$.

Proof. (1) By Theorem A.1, there is a constant $T(n, \Lambda)>0$ such that the conclusion is true for $S<T(n, \Lambda)$. For $a T_{h_{0}}>S \geq T(n, \Lambda)$, this follows directly from the $C^{0}$ bounds in Theorem 2.3(1) and Theorem A.2. The bounds also follow from the estimates in [11] and the $C^{0}$ estimates.

(2) also follows from Theorem 2.3 (2) and Theorem A.2.

\section{GENERALIZATIONS}

In this section, we will generalize some of the results Theorems 2.2 , 2.3, 2.4 without assuming that $g_{0}$ has bounded curvature. Let us introduce more spaces. Let $\left(M^{n}, h_{0}\right)$ be a complete noncompact Kähler manifold with bounded curvature. Let $0<a<b$ be constants. Let $\mathrm{Cl}\left(\mathcal{G}\left(h_{0} ; a, b\right)\right)$ be the set all continuous Hermitian metrics $g_{0}$ on $M$ such that there exist a sequence $g_{i} \in \mathcal{G}\left(h_{0} ; a, b\right)$ with $g_{i} \rightarrow g_{0}$ uniformly on $M$. Namely, $\sup _{M}\left|g_{i}-g_{0}\right|_{h_{0}} \rightarrow 0$ as $i \rightarrow \infty$. Let $\mathrm{Cl}_{\text {loc }}\left(\mathcal{G}\left(h_{0} ; a, b\right)\right)$ be the set all continuous Hermitian metrics $g_{0}$ on $M$ such that there exist a sequence $g_{i} \in \mathcal{G}\left(h_{0} ; a, b\right)$ with $g_{i} \rightarrow g_{0}$ uniformly on compact sets in $M$. For $\Lambda>0$, one can define $\mathrm{Cl}_{\text {loc }}\left(\mathcal{G}\left(h_{0} ; a, b, \Lambda\right)\right)$ similarly.

Theorem 3.1. Let $\left(M, h_{0}\right)$ be a complete noncompact Kähler manifold with bounded curvature and let $0<a<b$ be constants.

(1) Given any $g_{0} \in C l_{\text {loc }}\left(\mathcal{G}\left(h_{0} ; a, b\right)\right)$, there is a solution $g(t)$ to the Kähler-Ricci flow (1.1) on $M \times\left(0, \frac{a}{n} T_{h_{0}}\right)$ such that 
(i) $g(t)$ is Kähler for $t \in\left(0, \frac{a}{n} T_{h_{0}}\right)$ and $g(t) \rightarrow g_{0}$ as $t \rightarrow 0$ uniformly on compact sets of $M$;

(ii) for any $0<S<\frac{a}{n} T_{h_{0}}$, there is a constant $C>0$ such that

$$
C^{-1} h_{0} \leq g(t) \leq C h_{0}
$$

on $M \times[0, S]$

(iii) for any $0<s<S<\frac{a}{n} T_{h_{0}}$, and for any $l \geq 0$, we have

$$
\left|\nabla_{g(t)}^{l} \operatorname{Rm}(g(t))\right|_{g(t)}^{2} \leq \frac{C}{t^{l+2}}
$$

(2) If $g_{0} \in C l_{l o c}\left(\mathcal{G}\left(h_{0} ; a, b, \Lambda\right)\right)$ for some $\Lambda>0$, then the conclusion in (1) holds with $\frac{a}{n} T_{h_{0}}$ replaced by $a T_{h_{0}}$.

(3) If $g_{0} \in C l\left(\mathcal{G}\left(h_{0} ; a, b\right)\right)$, then the conclusion in (1) holds with $\frac{a}{n} T_{h_{0}}$ replaced by $a T_{h_{0}}$.

In particular, if $T_{h_{0}}=\infty$, then $g(t)$ above exists for all time $t>0$ such that the estimates above hold.

Proof. We first prove (1). By Theorem 4.2 in [1], there is some $T>0$ such that the conclusions in (1) hold when replacing $\frac{a}{n} T_{h_{0}}$ with $T$. Assume that $T$ is maximal such that this is true. By the proof of Lemma 3.1 in [1], there are continuous functions $A(t), B(t)$ on $[0, T)$ such that $A(t) h_{0} \leq g(t) \leq B(t) h_{0}$ with $A(t) \rightarrow \frac{a}{n}$ as $t \rightarrow 0$. Applying Theorem 2.4 (1) to $g(\epsilon)$ for small $\epsilon$ implies that $T \geq \epsilon+A(\epsilon) T_{h_{0}}$. We conclude that $T \geq \frac{a}{n} T_{h_{0}}$ by letting $\epsilon \rightarrow 0$.

We now prove part (2). Let $\left\{g_{i, 0}\right\}_{i=1}^{\infty} \subset \mathcal{G}\left(h_{0} ; a, b, \Lambda\right)$ such that $g_{i, 0} \rightarrow$ $g_{0}$ uniformly in compact sets of $M$. Then by Theorem 2.3 (2) and Theorem 2.4 (2), we have a sequence of solutions $g_{i}(t)$ to (1.1) with initial data $g_{i, 0}$ on $M \times\left(0, a T_{h_{0}}\right)$ converging to a smooth limit solution $g(t)$ to (1.1) on $M \times\left(0, a T_{h_{0}}\right)$. Moreover, for any $0<S<a T_{h_{0}}$, there is a constant $C>0$ independent of $i$ such that $C^{-1} h_{0} \leq g_{i}(t) \leq C h_{0}$ on $M \times(0, S]$, and thus there exists a smooth limit solution $g_{i}(t) \rightarrow g(t)$ on $M \times(0, S]$ satisfying (ii). From this we may prove that $g(t)$ satisfies (i) as in the proof of Theorem 4.1 in [1. Moreover by applying Theorem A.2 we may also conclude that $g(t)$ satisfies (iii).

To prove $(3)$, let $g_{0} \in \mathrm{Cl}\left(\mathcal{G}\left(h_{0} ; a, b\right)\right)$. By $(1)$, we obtain $g(t)$ satisfying the conclusions in (1). We need to prove that $g(t)$ can be extended up to $a T_{h_{0}}$ so that the estimates in (1) are true up to $a T_{h_{0}}$. By the proof of [1, Theorem 4.1], we have $g(t) \rightarrow g_{0}$ uniformly on $M$. Since $a h_{0} \leq g_{0} \leq b h_{0}$ and $g(t)$ has bounded curvature for $t>0$, by applying Theorem 2.2, 2.3, 2.4, the result follows. 
Corollary 3.1. Let $\left(M^{n}, h_{0}\right)$ be a complete noncompact Kähler manifold with bounded curvature. Suppose $g_{0}$ is another Kähler metric on $M$ such that (i) $a h_{0} \leq g_{0} \leq b h_{0}$ for some $0<a<b$; (ii) $\operatorname{Ric}\left(g_{0}\right) \geq-K g_{0}$ for some $K$; and (iii) there exist $r_{0}>0, p_{0}>n$ and a constant $C$ with

$$
\frac{1}{V_{g_{0}}\left(x, r_{0}\right)} \int_{B_{g_{0}}\left(x, r_{0}\right)}\left|\operatorname{Rm}\left(g_{0}\right)\right|^{p_{0}} \leq C
$$

for all $x \in M$. Then there is a solution $g(t)$ to the Kähler-Ricci flow with $g(0)=g_{0}$ on $M \times\left[0, a T_{h_{0}}\right)$ such that for any $0<S<a T_{h_{0}}$ there is a constant $C$ with $C^{-1} h_{0} \leq g(t) \leq C h_{0}$ on $M \times[0, S]$.

Proof. By the results in [10, 4, there is a short time solution $g(t)$ to the Kähler-Ricci flow (1.1) with $g(0)=g_{0}$. Moreover, the curvature has the following bound:

$$
|\operatorname{Rm}(g(t))| \leq C_{1} t^{-\frac{n}{p_{0}}}
$$

for some constant $C_{1}$. Since $p_{0}>n$, we have $g(t) \rightarrow g_{0}$ as $t \rightarrow 0$ uniformly on $M$. One can then apply Theorem 3.1 to conclude that the corollary is true.

\section{Appendix A.}

In this appendix we collect some known results for easy reference. The first result the basic existence theorem of W.-X. Shi [8]:

Theorem A.1. Suppose $g_{0}$ is a complete Kähler metric on a noncompact complex manifold $M^{n}$ with complex dimension $n$ with curvature bounded by a constant $k_{0}$. Then there exists $0<T \leq \infty$ depending only on $k_{0}$ and the dimension $n$, and a smooth solution $g(t)$ to (1.1) on $M \times[0, T)$ with initial condition $g(0)=g_{0}$ such that

(i) $g(t)$ has uniformly bounded curvature on $M \times\left[0, T^{\prime}\right]$ for all $0<$ $T^{\prime}<T$. More generally, for any $l \geq 0$ there exists a constant $C_{l}$ depending only on $l, k_{0}, n, T^{\prime}$ such that

$$
\sup _{M}\left|\nabla^{l} \operatorname{Rm}(g(t))\right|_{g(t)}^{2} \leq \frac{C_{l}}{t^{l}}
$$

on $M \times\left[0, T^{\prime}\right]$.

(ii) If $T<\infty$ and $\lim _{t \rightarrow T} \sup _{M}|R m(x, t)|<\infty$, then the $g(t)$ can be extended, as a solution to (1.1), beyond $T$ to $T_{1}>T$ so that (i) is still true with $T$ replaced by $T_{1}$.

The following is an immediate consequence of the theorem:

Corollary A.1. Let $g(t)$ be as in Theorem A.1. Then 
(i) $g(t)$ is Kähler and equivalent to $g_{0}$ for all $t \in[0, T)$, namely for all $T^{\prime}<T$, there a constant $C>0$ depending only on $k_{0}, n, T^{\prime}$ such that

$$
C^{-1} g_{0} \leq g(t) \leq C g_{0}
$$

on $M \times\left[0, T^{\prime}\right]$

(ii) There is a continuous function $\epsilon(t)>0$ depending only on $n, k_{0}$ with $\epsilon(t) \rightarrow 0$ as $t \rightarrow 0$ such that

$$
(1+\epsilon(t))^{-1} g_{0} \leq g(t) \leq(1+\epsilon(t)) g_{0} .
$$

Using Evan-Krylov theory [3, 5] or using maximum principle for Kähler-Ricci flow by Sherman-Weinkove [7], we have the following (see [1]):

Theorem A.2. Let $\left(M^{n}, h_{0}\right)$ be a complete noncompact Kähler manifold with bounded geometry of infinite order. Let $g(t)$ be a solution of the Kähler-Ricci flow (1.1) on $M \times[0, T)$ with initial data $g_{0}$ which is a complete Kähler metric. For any $x \in M$, suppose there is a constant $N>0$, such that

$$
N^{-1} h_{0} \leq g(t) \leq N h_{0}
$$

on $B_{h_{0}}(x, 1) \times[0, T)$. Then

(i)

$$
\left|\nabla_{h_{0}}^{k} g(t)\right|_{h_{0}}^{2} \leq \frac{C_{k}}{t^{k}}
$$

on $B_{h_{0}}(x, 1 / 2) \times(0, T)$, for some constant $C_{k}$ depending only on $k, n, T$ and $N$, and the bounds of $\left|\nabla^{l} \operatorname{Rm}\left(h_{0}\right)\right|_{h_{0}}$.

(ii) If we assume $\left|\nabla_{h_{0}}^{k} g_{0}\right|_{h_{0}}^{2}$ is bounded in $B_{h_{0}}(x, 1)$ by $c_{k}$, for $k \geq 1$, then

$$
\left|\nabla_{h_{0}}^{k} g(t)\right|_{h_{0}}^{2} \leq C_{k}
$$

on $B_{h_{0}}(x, 1 / 2) \times[0, T)$ for some constant $C_{k}$ depending only on $k, c_{k}, n, T$ and $N$.

We also need the following uniqueness theorem on Kähler-Ricci flow [2]:

Theorem A.3. Let $\left(M^{n}, h_{0}\right)$ be a complete noncompact Kähler manifold with bounded curvature. Let $g_{1}(x, t)$ and $g_{2}(x, t)$ be two solutions of the Kähler-Ricci flow (1.1) on $M \times[0, T]$ with the same initial data $g_{0}(x)=g_{1}(x, 0)=g_{2}(x, 0)$. Suppose there is a constant $C$ such that:

$$
C^{-1} h_{0} \leq g_{1}(t), g_{2}(t) \leq C h_{0}
$$

on $M \times[0, T]$. Then $g_{1} \equiv g_{2}$ on $M \times[0, T]$. 


\section{REFERENCES}

[1] Chau, A.; Li, K.-F.; Tam, L.-F., Deforming complete Hermitian metrics with unbounded curvature, arXiv:1402.6722, to appear in the Asian Journal of Mathematics.

[2] Chau, A.; Li, K.-F.; Tam, L.-F., Longtime existence of the Kähler-Ricci flow on $\mathbb{C}^{n}$, arXiv:1409.1906, to appear in the Transactions of the AMS.

[3] L.C. Evans, Classical solutions of fully nonlinear, convex, second order elliptic equations, Comm. Pure Appl. Math, 35 (1982), 333-363.

[4] Huang, S.; Tam, L.-F.,Kähler-Ricci flow with unbounded curvature, preprint, arXiv:1506.00322

[5] N.V. Krylov, Boundedly nonhomogeneous elliptic and parabolic equations, Izvestia Akad. Nauk. SSSR 46 (1982), 487-523. English translation in Math. USSR Izv. 20 (1983), no. 3, 459-492.

[6] Lott, J.; Zhang, Z., Ricci flow on quasi-projective manifolds, Duke Math. J., Volume 156, Number 1 (2011), 87-123.

[7] M. Sherman; B. Weinkove, Interior derivative estimates for the Kähler-Ricci flow, Pacific Journal of Mathematics, 257(2) (2012), 491-501.

[8] W.,-X., Shi, Ricci Flow and the uniformization on complete non compact Kähler manifolds, J. of Differential Geometry 45 (1997), 94-220.

[9] G. Tian and Z. Zhang, On the Kähler-Ricci flow on projective manifolds of general type, Chinese Ann. of Math. Ser. B 27 (2006), 179192.

[10] Xu, G.,Short-time existence of the Ricci flow on noncompact Riemannian manifolds, Trans. Amer. Math. Soc. 365 (2013), no. 11, 5605-5654.

[11] Yu, C.-J., A note on Kähler-Ricci flow, Math. Z. 272 (2012), no. 1-2, 191201.

Department of Mathematics, The University of British Columbia, Room 121, 1984 Mathematics Road, Vancouver, B.C., Canada V6T 1Z2

E-mail address: chau@math.ubc.ca

Department of Mathematics, The University of British Columbia, Room 121, 1984 Mathematics Road, Vancouver, B.C., Canada V6T $1 Z 2$

E-mail address: kfli@math.ubc.ca

The Institute of Mathematical Sciences and Department of Mathematics, The Chinese University of Hong Kong, Shatin, Hong Kong, CHINA.

E-mail address: Iftam@math.cuhk.edu.hk 